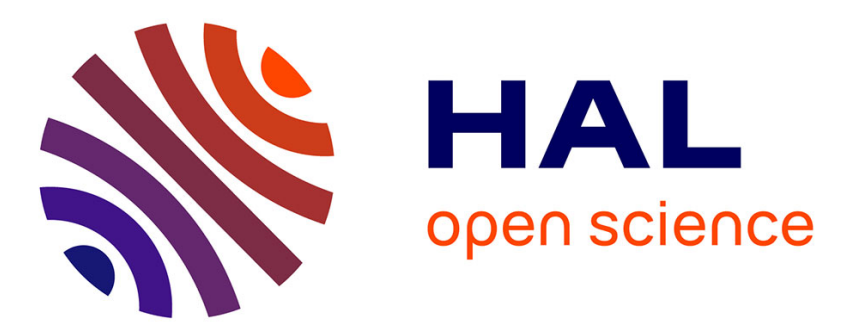

\title{
Evolution of phases in a shape memory alloy by local and global minimisations of a non convex potentials
}

Olivier Maisonneuve, Stéphane Pagano, Pierre Alart, Frédéric Lebon

\section{To cite this version:}

Olivier Maisonneuve, Stéphane Pagano, Pierre Alart, Frédéric Lebon. Evolution of phases in a shape memory alloy by local and global minimisations of a non convex potentials. IUTAM Symposium on Variations of Domain and Free-Boundary Problems in Solid Mechanics, 1999, Paris, France. pp.9-16, 10.1007/978-94-011-4738-5_2. hal-01832737

\section{HAL Id: hal-01832737 https://hal.science/hal-01832737}

Submitted on 8 Jul 2018

HAL is a multi-disciplinary open access archive for the deposit and dissemination of scientific research documents, whether they are published or not. The documents may come from teaching and research institutions in France or abroad, or from public or private research centers.
L'archive ouverte pluridisciplinaire HAL, est destinée au dépôt et à la diffusion de documents scientifiques de niveau recherche, publiés ou non, émanant des établissements d'enseignement et de recherche français ou étrangers, des laboratoires publics ou privés. 


\title{
EVOLUTION OF PHASES IN A SHAPE MEMORY ALLOY BY LOCAL AND GLOBAL MINIMISATIONS OF A NON CONVEX POTENTIALS
}

\author{
O. MAISONNEUVE, S. PAGANO, P. ALART AND F. LEBON \\ LMGC Université de Montpellier II - CNRS \\ UMR 5508 Place Eugène Bataillon \\ 34095 Montpellier Cedex 05
}

\section{Introduction}

A Shape Memory Alloy (SMA) is a mixture of martensite and austenite or only martensite or austenite. Austenite-martensite phase transformation leads to variations of composition. This transformation can appear by mechanical or thermal action. The characteristic pseudo-elastic behaviour appears from this modification in the material over certains conditions for which observe an hysteresis loop on the stress-strain relationship. This hysteresis can be influenced by several factors : thermal effect, dissipation, structural effect, existence of geometrical defect, etc..

Here, we use a model taking the temperature, the deformation and the volumic proportions of austenite and martensite as state variables. We adopt, here, Fremond's modelling in the non dissipative case which gives a non monotonic stress-strain relationship. Some experiments (Chrysochoos et al., 1995) show that during room temperature test, dissipative effects are strongly dominated by effects resulting from temperature variations of material associated to the latent heat. In our case, after elimination of the volumic proportions of martensite, the bulk energy density is a non convex function.

For a given problem, it is classical to look for the mechanical equilibrium by a global minimization of the "total" energy (potential energy). Moreover, the energy associated at each problem is not quasiconvex. Existence of absolute minima is not garanteed in all the case. It is possible to pose displacement boundary-value problems for which the total energy has no minimizer and then no absolute minimum ; the minimizing sequences are produced by displacements with fine-scale irregularities. Consequently, we 
introduce two relaxation procedures (convexification and quasiconvexification) for which existence of minimizer is garanteed in order to compare in the three cases, displacement solutions and evolutions of phases. The algorithm to find global minimum is based on an augmented Lagrangian method (decomposition-coordination method).

From a mechanical point of view, there is no reason to exclude local minima as possible equilibrium states. So, the evolution of phases may be different from the loading process to the unloading one. In order to fine local minima, we write the non convex potential as a difference of two convex functions (D.C.). Then we can formulate a problem of two variational inequalities in term of displacements and stresses. In the finite dimensional case, this problem is equivalent to search solutions which locally minimize the "total" energy. By applying partial convex duality, an inf-inf problem can be formulated with respect to an appropriately defined Lagrangian function.

We present a theoretical and numerical comparison between these two points of view in focusing out the evolution of phases.

\section{Constitutive law}

Here, we adopt the Fremond modelling (Fremond, 1987) with only two variants of martensite, and we denote by $\beta_{1}$ and $\beta_{2}$ their volumic proportions. We assume that the volumic free energies of the phases (respectively the austenitic one and the two martensitic ones) have the following form involving the small strain tensor $\mathbf{e}$,

$$
\begin{aligned}
& W_{0}(\mathbf{e}, T)=\frac{1}{2} \mathbf{e K e}-c(T)-C_{0} T \log T \\
& W_{-}(\mathbf{e}, T)=W_{0}(\mathbf{e}, T)+\alpha(T) \tau: \mathbf{e}+c(T) \\
& W_{+}(\mathbf{e}, T)=W_{0}(\mathbf{e}, T)-\alpha(T) \tau: \mathbf{e}+c(T)
\end{aligned}
$$

with $\alpha(T)=\left\{\begin{array}{ll}-a\left(T-T_{c}\right) & \text { if } T \leq T_{c} \\ 0 & \text { otherwise }\end{array}\right.$ and $c(T)=\frac{L}{T_{0}}\left(T-T_{0}\right)$

The phases are assumed to have the same elastic tensor $\mathbf{K}$, and the same heat capacity $\mathrm{C}$. The austenite-martensite phase transformation is associated with a residual stress tensor $\alpha \tau$ which is be chosen identically for the two variants. $L$ is the latent heat of phase transformation. Fremond writes the total volumic free energy $\Psi$ as a convex combination of the three energies: the volumic proportions are constrained to stay in the simplex C via the addition of the indicator function of this simplex :

$$
\begin{aligned}
\Psi\left(\mathbf{e}, T, \beta_{1}, \beta_{2}\right) & =\beta_{1} W_{-}(\mathbf{e}, T)+\beta_{2} W_{+}(\mathbf{e}, T) \\
& +\left(1-\beta_{1}-\beta_{2}\right) W_{0}(\mathbf{e}, T)+T I_{\mathcal{C}}\left(\beta_{1}, \beta_{2}\right)
\end{aligned}
$$

where $I_{\mathcal{C}}\left(\beta_{1}, \beta_{2}\right)$ is the indicator function of the convex set : 


$$
\mathcal{C}=\left\{\left(\gamma_{1}, \gamma_{2}\right) \quad \mid \quad 0 \leq \gamma_{i} \leq 1 \quad ; \quad \gamma_{1}+\gamma_{2} \leq 1\right\}
$$

We introduce the formalism of generalized standard materials and we neglect dissipation (Chrysochoos et al., 1995): we can eliminate the internal variables $\left(\beta_{1}, \beta_{2}\right)$. Then, a new potential can be defined as the minimum of three convex functions. Note that this potential is not convex:

$$
\begin{aligned}
W(\mathbf{e}, T) & :=\min \left\{\Psi\left(\mathbf{e}, T, \beta_{1}, \beta_{2}\right),\left(\beta_{1}, \beta_{2}\right) \in C\right\} \\
& =\min \left\{W_{0}(\mathbf{e}, T), W_{-}(\mathbf{e}, T), W_{+}(\mathbf{e}, T)\right\}
\end{aligned}
$$

With the three-well energy $W$, which does not depend on $\beta_{1}, \beta_{2}$, the material constitutive law is now written :

$$
\sigma \in \bar{\partial}_{\mathbf{e}} W(\mathbf{e}(\mathbf{u}), T)
$$

where $\bar{\partial}$ denotes the subdifferential operator of Clarke.

We consider an extension test and we represent the energy $W$ at different temperatures in Figure 2 : low temperature $T \leq T_{0}$, intermediate temperature $T_{0} \leq T \leq T_{c}$ and high temperature $T \geq T_{c}$.

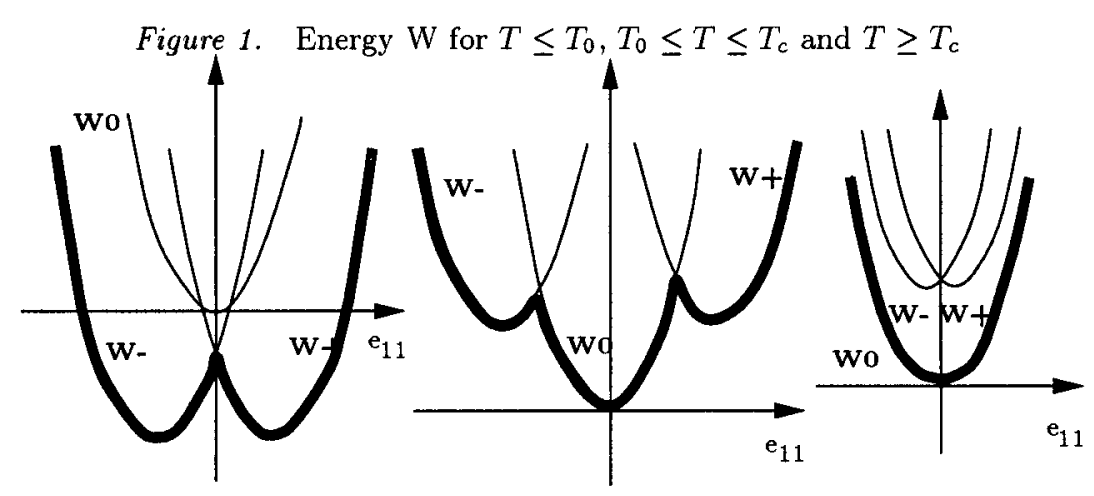

With the constitutive law (1), we etablish a classical formulation of the mechanical problem.

\section{Mechanical problem at fixed temperature}

Consider a boundary problem on a domain $\Omega$ of boundary $\Gamma$. Let $\mathbf{f}$ denote the density of body force applied on $\Omega, F$ the density of surfacic force applied on the part $\Gamma_{0}$ of $\Gamma$, and $U$ a displacement given on the complementary part $\Gamma_{1}$.

The mechanical problem at fixed temperature consists in finding the displacement field $\mathbf{u}$ and the stress field $\sigma$ verifying the following equations 


$$
\begin{array}{rc}
\operatorname{div} \sigma+\mathbf{f}=\mathbf{0} & \text { in } \Omega \\
\mathbf{e}(\mathbf{u})=\nabla_{\mathbf{s}} \mathbf{u} & \text { in } \Omega \\
\text { Constitutive law }: \sigma \in \bar{\partial}_{\mathbf{e}} W(\mathbf{e}(\mathbf{u})) & \text { in } \Omega \\
\mathbf{u}=\mathrm{U} & \text { on } \Gamma_{0} \\
\sigma . \mathbf{n}=\mathbf{F} & \text { on } \Gamma_{1}
\end{array}
$$

In the following, the set of kinematically admissible displacement fields with finite energy of the structure will be defined by :

$\mathbf{U}_{a d}=\left\{\mathbf{v} \in\left[\mathrm{H}^{1}(\Omega)\right]^{3}, \mathbf{v}=\mathbf{U}\right.$ on $\left.\Gamma_{0}\right\}$ and the stress spaces $\mathbf{H}=\left[\mathrm{L}^{2}(\Omega)\right]^{6}$.

\section{Global minimum}

\subsection{THE THREE-WELL ENERGY}

At first, we search for a global minimum of the three-well potential energy. To solve this problem, we consider an augmented Lagrangian. It is a function of a multiplier $\mu$ and of two independent variables $\mathbf{v}, \mathbf{q}$ corresponding to the displacement and strain fields.

$\mathrm{L}_{\mathbf{r}}(\mathbf{v}, \mathbf{q}, \mu)=\int_{\Omega} W(\mathbf{q}) d x-l(\mathbf{v})+\int_{\Omega} \mu:(\mathbf{e}(\mathbf{v})-\mathbf{q}) d x+\frac{r}{2} \int_{\Omega}((\mathbf{e}(\mathbf{v})-\mathbf{q}))^{2} d x$

with $l(\mathbf{v})=\int_{\Omega} \mathbf{f . v} d \Omega+\int_{\Gamma_{1}} \mathbf{F} . \mathbf{v} d \Gamma$ and $r \geq 0$.

We extend this non-convex density energy, the classical strategy of searching for the saddle points of the augmented Lagrangian i.e. the ( $u, p, \lambda)$ solutions of the problem :

$$
\mathrm{L}_{\mathbf{r}}(\mathbf{u}, \mathbf{p}, \mu) \leq \mathrm{L}_{\mathbf{r}}(\mathbf{u}, \mathbf{p}, \lambda) \leq \mathrm{L}_{\mathbf{r}}(\mathbf{v}, \mathbf{q}, \lambda), \forall v \in \mathbf{U}_{a d}, \forall \mathbf{q} \in \mathbf{H}, \forall \mu \in \mathbf{H}
$$

To solve the initial problem, we use an algorithm inspired of by Uzawa method (LeTallec et al., 1989) which involves a two-step iteration : a linear global problem and a non linear local problem.

\subsection{THE "RELAXED" ENERGY}

To profit by an existence theorem, we calculate the quasiconvexification QW of the previous potential by using the approach of Pipkin (Pipkin, 1990 ), and the convexification $\mathrm{CW}$ of $\mathrm{W}$. To determine the quasiconvexification, we take a unit cube B, we use the definition of QW (Pipkin, 1990)

$$
Q W(\mathbf{e}):=\liminf _{\mathbf{u}_{N} \rightarrow 0 \text { uniformly }} \int_{B} W\left(\mathbf{e}+\nabla_{s} \mathbf{u}_{N}\right) d x,
$$


and consider a sequence of displacement fields $\mathbf{u}_{N}$ for which the strain varies rapidly and periodically between two alternate constant values $e_{1}$ and $e_{2}$. The details of the calculus are given in the paper (Pagano et al., to appear). $\mathbf{u}_{N}$ is a periodic function of the variable $\mathbf{n} . \mathbf{x}$, of period $\frac{1}{N}$.

Pipkin defines this sequence on one period $\left[0, \frac{1}{N}\right]$ by

$$
\begin{gathered}
\mathbf{u}_{N}:= \begin{cases}2 \theta(\mathbf{n} \cdot \mathbf{x}) \mathbf{u} & \text { on }\left[0, \frac{1-\theta}{N}\right] \\
-2(1-\theta)\left(\mathbf{n} \cdot \mathbf{x}-\frac{1}{N}\right) \mathbf{u} & \text { on }\left[\frac{1-\theta}{N}, \frac{1}{N}\right]\end{cases} \\
\text { and } \mathbf{e}+\nabla_{s} \mathbf{u}_{N}=\left\{\begin{array}{l}
e_{1}=e+\theta(\mathbf{u} \otimes \mathbf{n}+\mathbf{n} \otimes \mathbf{u}) \\
e_{2}=e+(1-\theta)(\mathbf{u} \otimes \mathbf{n}+\mathbf{n} \otimes \mathbf{u})
\end{array}\right.
\end{gathered}
$$

where $\mathbf{u}$ and $\mathbf{n}$ are two constant vectors and $0<\theta<1$. We can prove that the density energy $\mathrm{QW}$ is :

$$
Q W(\mathbf{e})=\min _{\mathbf{u}, \mathbf{n}, 0 \leq \theta \leq 1}\left[(1-\theta) W\left(\mathbf{e}_{1}\right)+\theta W\left(\mathbf{e}_{2}\right)\right] .
$$

This minimization can be performed by discrimination of all the possible cases for $W\left(\mathbf{e}_{1}\right)$ and $W\left(\mathbf{e}_{2}\right)$. We obtain a similar form, noted RW, for the convexification and the quasiconvexification of the density energy $W$. for $c+\frac{\mathcal{G}}{2} \geq 0$ :

$$
R W(\mathbf{e})= \begin{cases}W_{-}(\mathbf{e}) & \text { if } \alpha \tau: \mathbf{e} \leq-c+\frac{\mathcal{G}}{2} \\ W_{0}(\mathbf{e})+\frac{1}{2 \mathcal{G}}\left(\alpha \tau: \mathbf{e}+c+\frac{\mathcal{G}}{2}\right)^{2} & \text { if }-c+\frac{\mathcal{G}}{2} \leq \alpha \tau: \mathbf{e} \leq-c-\frac{\mathcal{G}}{2} \\ W_{0}(\mathbf{e}) & \text { if } \alpha|\tau: \mathbf{e}| \leq c+\frac{\mathcal{G}}{2} \\ W_{0}(\mathbf{e})+\frac{1}{2 \mathcal{G}}\left(\alpha \tau: \mathbf{e}-c-\frac{\mathcal{G}}{2}\right)^{2} & \text { if } c+\frac{\mathcal{G}}{2} \leq \alpha \tau: \mathbf{e} \leq c-\frac{\mathcal{G}}{2} \\ W_{+}(\mathbf{e}) & \text { if } \alpha \tau: \mathbf{e} \geq c-\frac{\mathcal{G}}{2}\end{cases}
$$

The functional RW is equal to the convexification of $\mathrm{W}$ if $\mathcal{G}=\mathcal{G}_{c}$ and to the quasiconvexification for $\mathcal{G}=\mathcal{G}_{q}$ with :

$$
\begin{aligned}
\mathcal{G}_{c} & =2\left[\min _{\mathbf{E} \in M_{s}^{3 \times 3}} W_{-}(\mathbf{E})-c\right]=-\alpha^{2} \tau: \mathbf{K}^{-1} \tau \\
\text { and } \quad \mathcal{G}_{q} & =2\left[\min _{\mathbf{u}, \mathbf{n}} W_{-}(\mathbf{u} \otimes \mathbf{n}+\mathbf{n} \otimes \mathbf{u})-c\right]
\end{aligned}
$$

In particular, $\mathcal{G}_{q}=\mathcal{G}_{c}$ if $\tau$ has the form $\mathbf{a} \otimes \mathbf{b}+\mathbf{b} \otimes \mathbf{a}$ where $\mathbf{a}$ and $\mathbf{b}$ are two vectors. If we restrict our attention to the case of isotropic Hooke's law with Lamé coefficients $\lambda$ and $\mu$, and if we suppose that $\tau=\mathrm{I}$, we can calculate the coefficients $\mathcal{G}_{q}$ and $\mathcal{G}_{c}$ to obtain the following results :

$$
\mathcal{G}_{c}=\frac{-\alpha^{2}}{2 \lambda+\mu} \text { and } \mathcal{G}_{q}=\frac{-\alpha^{2}}{\lambda+2 \mu}
$$

The optimal values of $\theta$ which minimize (3), noted $\theta_{-}$and $\theta_{+}$may be interpreted as two internal variables which represent the "proportions of 
martensite" of the mixture. These values are the following :

$$
\left(\theta_{-}, \theta_{+}\right)= \begin{cases}(1,0) & \text { if } \alpha \tau: \mathbf{e} \leq-c+\frac{\mathcal{G}}{2} \\ \left(\frac{\alpha \tau: \mathbf{e}+c+\frac{\mathcal{G}}{2}}{\mathcal{G}}, 0\right) & \text { if }-c+\frac{\mathcal{G}}{2} \leq \alpha \tau: \mathbf{e} \leq-c-\frac{\mathcal{G}}{2} \\ (0,0) & \text { if } \alpha|\tau: \mathrm{e}| \leq c+\frac{\mathcal{G}}{2} \\ \left(0, \frac{-\alpha \tau: \mathbf{e}+c+\frac{\mathcal{G}}{2}}{\mathcal{G}}\right) & \text { if } c+\frac{\mathcal{G}}{2} \leq \alpha \tau: \mathbf{e} \leq c-\frac{\mathcal{G}}{2} \\ (0,1) & \text { if } \alpha \tau: \mathbf{e} \geq c-\frac{\mathcal{G}}{2}\end{cases}
$$

For the case $c+\frac{\mathcal{G}}{2} \leq 0$ we obtain, also, similar expressions for QW and CW (analogous to the Pipkin's result for a two-well energy). We see that $\mathrm{QW}$ and $\mathrm{CW}$ are piecewise quadratic and $C^{1}$. From a well known result of Dacarogna, any solution of this relaxed problem is a limit of a minimizing sequence of the potential associated to the initial problem (Dacorogna, 1985). To solve the relaxed problem, we use the same method as for the initial problem.

\section{Local minimum}

The potential $\mathrm{W}$, at fixed temperature, can appear as the difference of two convex functions (d.c.) (Pagano et al., to appear) $\varphi_{1}$ and $\varphi_{2}$ :

$$
\varphi_{1}(\mathbf{e})=W_{0}(\mathbf{e}) \quad \text { and } \quad \varphi_{2}(\mathbf{e})=\max (\alpha \mid \text { tre } \mid-c)
$$

Based on this decomposition, we formulate the mechanical problem as two variational inequalities. In the finite dimensional case, this problem is equivalent to finding local minima of the potential energy. The decomposition leads to the introduction of a Lagrangian $L_{\mathbb{I}}$, in the terminology of Auchmuty (1989). It depends on the displacement field corresponding to a stress field, and it is function of the energy $\Phi_{1}$ and the Fenchel convex conjugate function $\Phi_{2}^{*}$. This Lagrangian is separately convex in each of the dual variables, but the convexity of $L_{\mathbb{I}}$ is not guaranteed in general. An algorithm is derived to reach $\partial$-critical points of the Lagrangians $L_{\mathbb{I}}$. These points are defined as follows :

$\left(\mathbf{u}, \sigma_{2}\right) \in \mathbf{U}_{a d} \times \mathbf{H}$ is a $\partial$-critical point of $\mathrm{L}_{\mathbb{I}}$ if :

$$
\begin{aligned}
& \mathrm{L}_{\mathbb{I}}\left(\mathbf{u}, \sigma_{2}\right) \leq \mathrm{L}_{\mathbb{I}}\left(\mathbf{v}, \sigma_{2}\right), \quad \forall \mathbf{v} \in \mathbf{U}_{a d} \\
& \mathrm{~L}_{\mathbb{I}}\left(\mathbf{u}, \sigma_{2}\right) \leq \mathrm{L}_{\mathbb{I}}(\mathbf{u}, \tau), \quad \forall \tau \in \mathrm{H}
\end{aligned}
$$

The relationship between critical points of the potential energy and those of $\mathrm{L}_{\mathrm{II}}$ is somewhat more delicate. If $\left(\mathbf{u}, \sigma_{2}\right) \in \mathbf{U}_{a d} \times \mathbf{H}$ is a $\partial$-critical point of the Lagrangian then $\mathbf{u}$ is a critical point of the potential energy. 
This convex difference algorithm constructs minimizing sequence of $\mathrm{L}_{\mathrm{II}}$. It permits us to characterize a $\partial$-critical point of $\mathrm{L}_{\mathbb{I I}}$. In practice, it determines local minima of the Lagrangian. It is also split into two steps, (global and local steps).

We remark that the "relaxed" energy can, also, be split into the difference of two convex functions $\varphi_{1}$ and $R \varphi_{2}$. The same numerical method can be applied to the resolution of the relaxed problem.

$$
\left(R \varphi_{2}\right)= \begin{cases}-\alpha \tau: \mathbf{e}-c & \text { if } \alpha \tau: \mathbf{e} \leq-c+\frac{\mathcal{G}}{2} \\ \frac{-1}{2 \mathcal{G}}\left(\alpha \tau: \mathrm{e}+c+\frac{\mathcal{G}}{2}\right)^{2} & \text { if }-c+\frac{\mathcal{G}}{2} \leq \alpha \tau: \mathbf{e} \leq-c-\frac{\mathcal{G}}{2} \\ 0 & \text { if } \alpha|\tau: \mathrm{e}| \leq c+\frac{\mathcal{G}}{2} \\ \frac{-1}{2 \mathcal{G}}\left(\alpha \tau: \mathrm{e}-c-\frac{\mathcal{G}}{2}\right)^{2} & \text { if } c+\frac{\mathcal{G}}{2} \leq \alpha \tau: \mathbf{e} \leq c-\frac{\mathcal{G}}{2} \\ \alpha \tau: \mathrm{e}-c & \text { if } \alpha \tau: \mathbf{e} \geq c-\frac{\mathcal{G}}{2}\end{cases}
$$

\section{Numerical simulations at fixed temperature}

In this part, we compare the three points of view. We assume that $\tau=\mathbf{I}$ and we consider a flat sample with a geometrical defect submitted to a strain controlled loading-unloading process. For symmetry reasons, only a quarter of the sample is represented [Figure 3].

Figure 2. Flat sample
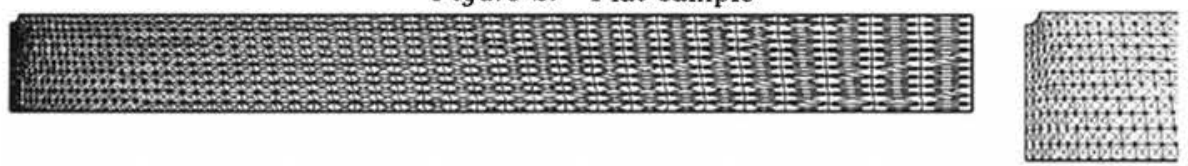

The incremental process is performed using loading steps. At each step the algorithm is initialised to the state at the end of the previous step.

With the initial energy $W$ and the D.C. approaches, we notice that a local non monotone strain-stress law (figure 1) leads to a monotone displacement-force diagram. Moreover a hysteresis behaviour is shown. For a given displacement value loading or unloading process, the phase transformation in the flat is not the same. The first picture in figure 4 represents the binary phases distribution in the flat. According to the chosen modelling, the proportions are either zero or one almost everywhere. Roughly speaking the phase transition is locally instantaneous.

With the global minimization, (quasiconvexified or not), the hysteresis vanishes. The distribution of phases, issuing from the minimisation of the quasiconvexification, appears as the "homogenization" of the phase distribution obtained for the non convex potential W. Some differences are obtained about the spatial repartition of phases for the quasiconvexification (figure 4-2) and the convexification (figure 4-3). With $\mathrm{CW}$, the zone 
where the volumic proportion of martensite lies between zero and one is more important than for relaxed energy QW.

Aknowledgement : We thank Doctor Licht for his very interesting and helpful comments.

\section{References}

Pipkin, A.C. (1990) Elastic material with two preferred states, Q. J. Mech: appl. Math.Vol. no 3,pp. 1-15

Pagano, S., Alart, P., Lebon, F. and Maisonneuve, O. (to appear) Evolution of phases in a shape memory alloys by local and global minimisations of a non convex potential

Fremond, M. (1987) Matériaux à mémoire de forme, R. Acad. Sci. ParisVol. no 304,pp. 239-244

LeTallec, P. and Vidrascu, M. (1989) Une méthode numérique pour les problèmes d'équilibre de corps hyperélastiques incompressibles en grandes déformations, $\mathrm{Nu}$ mer. Math.Vol. no 43,pp. 199-224

Dacorogna, F. (1989) Direct methods in the Calculus of Variations, Berlin : Springerverlag

Auchmuty, G. (1989) Duality algorithms for nonconvex variational principles, Num. Func. An. \& Opt.Vol. no 10,pp. 211-264

Chrysochoos, A., Pham, H., and Maisonneuve, O. (1995) Couplages thermomécanique du comportement d'alliages $\mathrm{Cu}-\mathrm{Zn}-\mathrm{Al}$ et $\mathrm{Ni}-\mathrm{Ti}$, C. R. Acad. Sci. ParisVol. no 320,pp. 217-223

Figure 3. Results with energies W(4-1), CW(4-2) and QW(4-3)
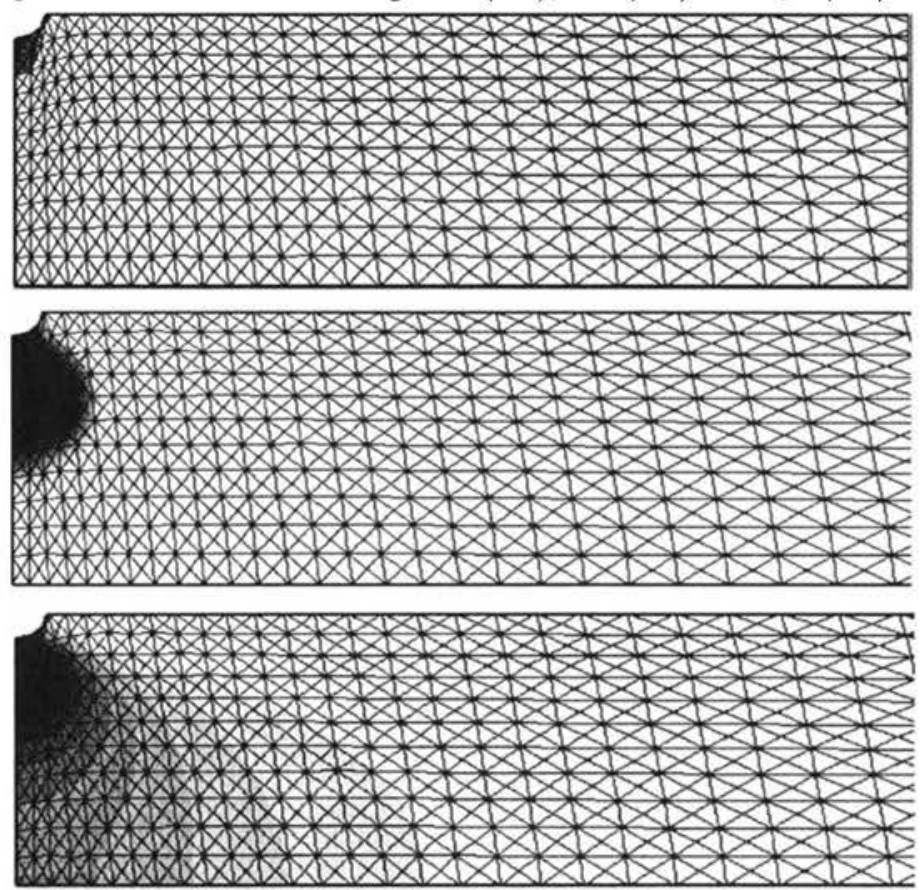